\title{
ANALYSIS OF CONVECTIVE-RADIATIVE POROUS FIN WITH TEMPERATURE-DEPENDENT INTERNAL HEAT GENERATION AND MAGNETIC FIELD USING HOMOTOPY PERTURBATION METHOD
}

\author{
Gbeminiyi Sobamowo \\ Department of Mechanical Engineering, University of Lagos, Akoka, Lagos, Nigeria \\ mikegbeminiyi@gmail.com \\ Olurotimi Adeleye \\ Department of System Engineering, University of Lagos, Akoka, Lagos, Nigeria \\ rotimiadeleye1711@gmail.com \\ Ahmed Yinusa \\ Department of Mechanical Engineering, University of Lagos, Akoka, Lagos, Nigeria \\ mynotebook2010@yahoo.com
}

[Received: September 1, 2017; Accepted: November 15, 2017]

\begin{abstract}
In this work, thermal behavior of convective-radiative porous fin with temperature-dependent internal heat generation subjected to magnetic field using homotopy perturbation method is analyzed. The developed symbolic heat transfer model is used to investigate the effects of convective, radiative, magnetic parameters on the thermal performance of the porous fin. From the study, it is established that increase in porosity, convective, radiative and magnetic parameters increase the rate of heat transfer from the fin and consequently, improve the efficiency of the fin. The homotopy perturbation method used in the work is validated with the results of a numerical method. The results of the two methods are in excellent agreement. Therefore, this study provides a platform for comparison of results of any other method of analysis of the problem. Also, such an analytical tool is valuable as a design and optimization approach for finned heat exchangers where each fin/row is analytically analyzed and where the surrounding fluid is influenced by a magnetic field.
\end{abstract}

Mathematical Subject Classification: 05C38, 15A15

Keywords: Thermal analysis, porous fin, convective-radiative fin, magnetic field, homotopy perturbation method

\section{INTRODUCTION}

The continuous demands for high performance thermal equipment have been part of the driving forces behind the present advancements in thermal technology. However, the production of excess heat is inevitable during the operation of thermal equipment. If such generated excessive heat levels are not controlled or dissipated, it leads to thermal-induced failure in the thermal systems. Consequently, the need for fins or 
extended surfaces as heat transfer enhancers in the thermal systems is indispensable. The applications of the extended surfaces in various systems such as electronic and microelectronics components, high-power semi-conductor devices, high-power lasers, light emitting diodes (LEDs), computer cooling, sensitive devices etc. have attracted various research interests in past decades. Also, the enhancement of heat transfer from thermal equipment and component through the use of porous fin has been a subject of research in recent times following the pioneer work of Kiwan and Al-Nimr [1]. In their analysis, the developed nonlinear model was solved numerically while Kiwan 2 2, 4] developed a simple method to study the performance of porous fins in a natural convection environment. Also, Gorla and Bakier [5] used a numerical approach to study the thermal performance of a convective-radiative rectangular porous fin. Kundu and Bhanja [6] and Kundu et al. [7] presented an analytical model for the optimization of porous fins and computation of maximum heat transfer in porous fins. With the help of a finite difference scheme, Taklifi et al. [8] investigated the effects of magnetohydrodynamics (MHD) on the performance of a rectangular porous fin. Bhanja and Kundu [9] and Kundu et al. 10] applied Adomian decomposition method to analytically investigate thermal analysis of a constructal T-shape porous fin with radiation effects and to analyze the optimum design of porous fin of various profiles operating in convection environment, respectively. In another work, Gorla et al. 11 applied Spectral collocation method (SCM) to study the effects of variable thermal conductivity on the natural convection and radiation in porous fin. Saedodin and Shahababaei 12 adopted the homotopoy perturbation method (HPM) to analyze heat transfer in longitudinal porous fins while Darvishi et al. 13] and Moradi et al. [14] and Ha et al. 15 utilized homotopy analysis method (HAM) to provide solutions to the natural convection and radiation in a porous and porous moving fins. Also, Hoshyar et al. 16 used the homotopy perturbation method and collocation method (CM) and presented thermal performance analysis of porous fins with temperaturedependent heat generation. The least square method (LSM) was used by Hatami and Ganji 17,18$]$ to study the thermal behaviour of convective-radiative in porous fin with different sections and ceramic materials. Recently, Rostamiyaan et al. 19 applied the variational iterative method (VIM) to develop analytical solution for heat transfer in porous fins while Ghasemi et al. 20 used differential transformation method (DTM) for heat transfer analysis in porous and solid fin.

The previous research works on the thermal analysis of fin have been generally carried out using ADM, HAM, VIM, DTM etc. However, the determination of Adomian polynomials as carried out in ADM, the need for small perturbation parameter as required in traditional PMs, and the rigour of the derivations of differential transformations or recursive relation as carried out in DTM have limited the applications of these method to wider varieties of applications. Moreover, the lack of rigorous theories or proper guidance for choosing initial approximation, auxiliary linear operators, auxiliary functions, auxiliary parameters, and the requirements of conformity of the solution to the rule of coefficient ergodicity as done in HAM, the search Langrange multiplier as carried in VIM, and the challenges associated with proper construction of the approximating functions for arbitrary domains or geometry of interest as in the Galerkin weighted residual method (GWRM), least square method (LSM) 
and collocation method $(\mathrm{CM})$ are some of the difficulties in the method that are are not experienced by HPM. Furthermore, in the class of the newly developed approximate analytical methods, the homotopy perturbation method is considered to relatively simple with fewer requirements for mathematical rigour or skill. It is a total approximate analytic method unlike DTM, HAM, ADM, VIM etc. where the search for a particular value that will satisfy second the boundary condition necessitates use of a numerical method with the use of a software which could result in additional computational cost in the generation of solution to the problem. Also, the homotopy perturbation method eliminates the "the small parameter assumption" as carried in the traditional perturbation methods. It is a powerful method that gives acceptable analytical results with convenient convergence and stability $21-25]$. Therefore, in finding approximate analytical solutions to linear and nonlinear differential equations, HPM has fast gained ground and it appears in many engineering and scientific research papers. It is an approximate analytical method that could solve differential equations, difference equation, differential-difference equations, fractional differential equation, pantograph equation and integro-differential equation. It solves nonlinear integral and differential equations without linearization, discretization, closure, restrictive assumptions, perturbation, approximations, round-off error and discretization that could result in massive numerical computations. It does not require small parameters in algebraic or differential equations as done in the other traditional perturbation methods (regular and singular perturbation). It provides excellent approximations to the solution of non-linear equation with high accuracy.

To the best of the authors' knowledge, the analysis of heat transfer in convectiveradiative porous fin with temperature-dependent internal heat generation and subjected to magnetic field using homotopy perturbation method has not been carried out. Therefore, in this present study, the thermal behavior of convective-radiative porous fin with temperature-dependent internal heat generation and subjected to magnetic field is studied using the homotopy perturbation method. The developed symbolic thermal model is used to investigate the effects of convective, radiative, and magnetic parameters on the thermal performance of the porous fin.

\section{Problem formulation}

Consider a straight porous fin of length $L$ and thickness t exposed on both faces to a convective environment at temperature $T_{\infty}$. The fin is heated internally and subjected to a uniform magnetic field as shown in Figure 1. Assuming the porous medium is homogeneous, isotropic and saturated with a single phase fluid. The physical properties of solid as well as fluid are considered as constant except density variation of liquid, which may affect the buoyancy term where Boussinesq approximation is employed. Fluid and porous media are locally in thermodynamic equilibrium in the domain and surface radiative transfers and non-Darcian effects are negligible, the temperature inside the fin varies along the length only and remains constant with time, there is no thermal contact resistance at the fin base and the fin tip is adiabatic type. 


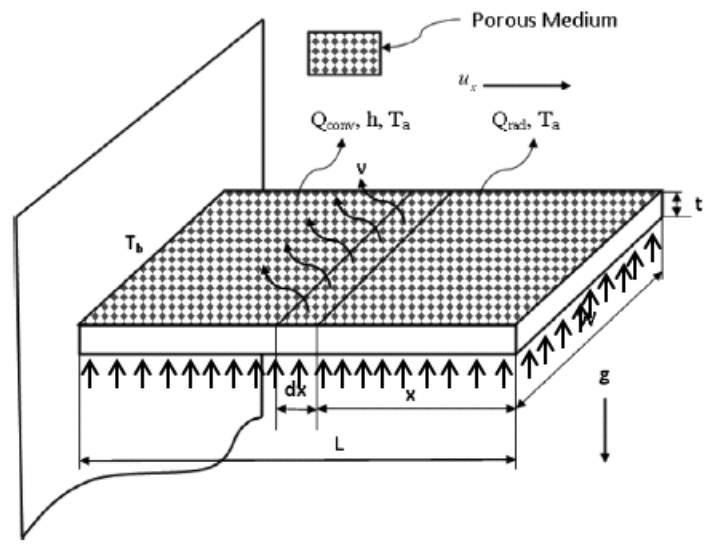

Magnetic field, $\mathbf{B}_{\mathbf{y}}$

Figure 1. Schematic of the convective-radiative longitudinal porous fin with magnetic field

Based on Darcy's model and following the above assumptions, the thermal energy balance in the fin could be expressed

$$
\begin{aligned}
q_{x}-\left(q_{x}+\frac{\delta q}{\delta x} d x\right)+q(T) d x=\dot{m} c_{p}\left(T-T_{a}\right) & +h P(1-\tilde{\varepsilon})\left(T-T_{a}\right) d x+ \\
+ & +\sigma \varepsilon P\left(T^{4}-T_{a}^{4}\right) d x+\frac{\mathbf{J}_{\mathbf{c}} \times \mathbf{J}_{\mathbf{c}}}{\sigma} d x,
\end{aligned}
$$

where

$$
\mathbf{J}_{\mathbf{c}}=\sigma_{e}(\mathbf{E}+\mathbf{V} \times \mathbf{B}) .
$$

The mass flow rate of the fluid passing through the porous material can be written as

$$
\dot{m}=\rho u(x) W d x .
$$

From Darcy's model

$$
u(x)=\frac{g K \beta}{v}\left(T-T_{a}\right) .
$$

Therefore, equation (1) becomes

$$
\begin{aligned}
q_{x}-\left(q_{x}+\frac{\delta q}{\delta x} d x\right) & +q(T) d x=\frac{\rho c_{p} g K \beta}{v}\left(T-T_{a}\right)^{2} d x+ \\
& +h P(1-\tilde{\varepsilon})\left(T-T_{a}\right) d x+\sigma \varepsilon P\left(T^{4}-T_{a}^{4}\right) d x+\frac{\mathbf{J}_{\mathbf{c}} \times \mathbf{J}_{\mathbf{c}}}{\sigma} d x .
\end{aligned}
$$

As $d x \rightarrow 0$, equation 5 reduces

$$
-\frac{d q}{d x}+q(T)=\frac{\rho c_{p} g K \beta}{v}\left(T-T_{a}\right)^{2}+h P(1-\tilde{\varepsilon})\left(T-T_{a}\right)+\sigma \varepsilon P\left(T^{4}-T_{a}^{4}\right)+\frac{\mathbf{J}_{\mathbf{c}} \times \mathbf{J}_{\mathbf{c}}}{\sigma} .
$$


From Fourier's law of heat conduction, the rate of heat conduction in the fin is given by

where

$$
q=-k_{\mathrm{eff}} A_{c r} \frac{d T}{d x}
$$

$$
k_{\text {eff }}=\phi k_{f}+(1-\phi) k_{s} .
$$

Following Rosseland diffusion approximation, the radiation heat transfer rate is

$$
q=-\frac{4 \sigma A_{c r}}{3 \beta_{R}} \frac{d T^{4}}{d x} .
$$

Substituting equation (9) into equation (6) we have

$$
\begin{aligned}
& \frac{d}{d x}\left(k_{\mathrm{eff}} A_{c r} \frac{d T}{d x}+\frac{4 \sigma A_{c r}}{3 \beta_{R}} \frac{d T^{4}}{d x}\right)+q(T)=\frac{\rho c_{p} g K \beta}{v}\left(T-T_{a}\right)^{2}+ \\
& +h P(1-\tilde{\varepsilon})\left(T-T_{a}\right)+\sigma \varepsilon P\left(T^{4}-T_{a}^{4}\right)+\frac{\mathbf{J}_{\mathbf{c}} \times \mathbf{J}_{\mathbf{c}}}{\sigma} .
\end{aligned}
$$

Further simplification of 10 gives the governing differential equation for the fin as

$$
\begin{aligned}
\frac{d^{2} T}{d x^{2}}+\frac{4 \sigma}{3 \beta_{R} k_{\mathrm{eff}}} \frac{d}{d x}\left(\frac{d T^{4}}{d x}\right)- & \frac{\rho c_{p} g K \beta}{k_{\mathrm{eff}} t v}\left(T-T_{a}\right)^{2}-\frac{h(1-\tilde{\varepsilon})}{k_{\mathrm{eff}} t}\left(T-T_{a}\right)- \\
& -\frac{\sigma \varepsilon}{k_{\mathrm{eff}} t}\left(T^{4}-T_{a}^{4}\right)-\frac{\mathbf{J}_{\mathbf{c}} \times \mathbf{J}_{\mathbf{c}}}{\sigma k_{\mathrm{eff}} A_{c r}}+\frac{q(T)}{k_{\mathrm{eff}} A_{c r}}=0 .
\end{aligned}
$$

The boundary conditions are

$$
\begin{array}{ll}
x=0, & \frac{d T}{d x}=0, \\
x=L, & T=T_{b} .
\end{array}
$$

But

$$
\frac{\mathbf{J}_{\mathbf{c}} \times \mathbf{J}_{\mathbf{c}}}{\sigma}=\sigma B_{o}^{2} u^{2}
$$

After substitution of equation (13) into equation (11),

$$
\begin{aligned}
\frac{d^{2} T}{d x^{2}}+\frac{4 \sigma}{3 \beta_{R} k_{\mathrm{eff}}} \frac{d}{d x}\left(\frac{d T^{4}}{d x}\right) & -\frac{\rho c_{p} g K \beta}{k_{\mathrm{eff}} t v}\left(T-T_{a}\right)^{2}-\frac{h(1-\tilde{\varepsilon})}{k_{\mathrm{eff}} t}\left(T-T_{a}\right)- \\
& -\sigma \varepsilon P\left(T^{4}-T_{a}^{4}\right)-\frac{\sigma B_{o}^{2} u^{2}}{k_{\mathrm{eff}} A_{c r}}\left(T-T_{a}\right)+\frac{q(T)}{k_{\mathrm{eff}}} A_{c r}=0
\end{aligned}
$$

The case considered in this work is a situation where small temperature difference exists within the material during the heat flow. This actually necessitated the use of temperature-invariant physical and thermal properties of the fin. Also, it has been established that under such scenario, the term $T^{4}$ can be expressed as a linear function of temperature. Therefore, we have

$$
T^{4}=T_{\infty}^{4}+4 T_{\infty}^{3}\left(T-T_{\infty}\right)+6 T_{\infty}^{2}\left(T-T_{\infty}\right)^{2}+\ldots \cong 4 T_{\infty}^{3} T-3 T_{\infty}^{4} .
$$

Also, it is given that

$$
q(T)=q_{0}\left[1+\lambda\left(T-T_{\infty}\right)\right]
$$


On substituting equation (15) into equation (14), we arrive at

$$
\begin{aligned}
\frac{d^{2} T}{d x^{2}}+\frac{16 \sigma}{3 \beta_{R} k_{\mathrm{eff}}} \frac{d^{2} T}{d x^{2}}- & \frac{\rho c_{p} g K \beta}{k_{\mathrm{eff}} t v}\left(T-T_{a}\right)^{2}-\frac{h(1-\tilde{\varepsilon})}{k_{\mathrm{eff}} t}\left(T-T_{a}\right)-4 \sigma \varepsilon P T_{a}^{3}\left(T-T_{a}\right)- \\
& -\frac{\sigma B_{o}^{2} u^{2}}{k_{\mathrm{eff}}} A_{c r}\left(T-T_{a}\right)+\frac{q_{o}}{k_{\mathrm{eff}}} A_{c r}\left[1+\lambda\left(T-T_{\infty}\right)\right]=0, \quad(16)
\end{aligned}
$$

On introducing the following dimensionless parameters in equation 17 into equation (16),

$$
\begin{gathered}
X=\frac{x}{L}, \quad \theta=\frac{T-T_{a}}{T_{b}-T_{a}}, \quad R a=\frac{g k \beta\left(T_{b}-T_{\infty}\right) b}{\alpha \nu k_{r}}, \\
N c^{2}=\frac{p b^{2} h}{k_{\mathrm{eff}} t}, \quad R d=\frac{4 \sigma_{s t} T_{\infty}^{3}}{3 \beta_{R} k_{\mathrm{eff}}}, \quad N r=\frac{4 \sigma_{s t} b T_{\infty}^{3}}{k_{\mathrm{eff}}}, \\
H=\frac{\sigma B_{0}^{2} u^{2}}{k_{\mathrm{eff}} A_{b}}, \quad Q=\frac{q_{o} t}{A_{c r} h\left(T_{b}-T_{a}\right)}
\end{gathered}
$$

we arrive at the dimensionless form of the governing equation $(16)$ as

$$
(1+4 R d) \frac{d^{2} \theta}{d X^{2}}-R a \theta^{2}-N c(1-\tilde{\varepsilon}) \theta-N r \theta-H \theta+N c^{2} Q(1+\gamma \theta)=0,
$$

or

$$
\begin{aligned}
\frac{d^{2} \theta}{d X^{2}}-\frac{R a}{1+4 R d} \theta^{2}-\frac{N c(1-\tilde{\varepsilon})}{1+4 R d} \theta-\frac{N r}{1+4 R d} \theta- & \\
& \quad-\frac{H}{1+4 R d} \theta+\frac{N c^{2} Q}{1+4 R d}(1+\gamma \theta)=0
\end{aligned}
$$

Equation 19 can be rewritten as

$$
\frac{d^{2} \theta}{d X^{2}}-S_{h} \theta^{2}-M_{a}^{2} \theta+M^{2} G(1+\gamma \theta)=0,
$$

where

$$
\begin{gathered}
S_{h}=\frac{R a}{(1+4 R d)}, \quad M^{2}=N c^{2}, \quad G=\frac{Q}{1+4 R d}, \\
M_{a}^{2}=\frac{N c(1-\tilde{\varepsilon})}{1+4 R d}+\frac{N r}{1+4 R d}+\frac{H}{1+4 R d} .
\end{gathered}
$$

The dimensionless boundary conditions are as follows:

$$
\begin{array}{cc}
X=0, & \frac{d \theta}{d X}=0 \\
X=1, & \theta=1
\end{array}
$$

\section{Method of Solution by homotopy perturbation method}

3.1. Choice of a solution method. It is very difficult to develop a closed-form solution for the above non-linear equation (19). Therefore, recourse has to be made to either an approximation analytical method, semi-numerical method or numerical method of solution. In this work, homotopy perturbation method is used to solve the equation. 
3.2. The basic idea of the homotopy perturbation method. In order to establish the basic idea behind homotopy perturbation method, consider a system of nonlinear differential equations given as

$$
A(U)-f(r)=0, \quad r \in \Omega
$$

with the boundary conditions

$$
B\left(u, \frac{\partial u}{\partial \eta}\right)=0, \quad r \in \Gamma
$$

where $A$ is a general differential operator, $B$ is a boundary operator, $f(r)$ is a known analytical function and $\Gamma$ is the boundary of the domain $\Omega$.

The operator $A$ can be divided into two parts, which are $L$ and $N$, where $L$ is a linear operator and $N$ is a non-linear operator. Equation 21 can, therefore, be rewritten as follows:

$$
L(u)+N(u)-f(r)=0 .
$$

By the homotopy technique, a homotopy $U(r, p): \Omega \times[0,1] \rightarrow R$ can be constructed, which satisfies the equation

$$
H(U, p)=(1-p)\left[L(U)-L\left(U_{o}\right)\right]+p[A(U)-f(r)]=0, \quad p \in[0,1]
$$

or

$$
H(U, p)=L(U)-L\left(U_{o}\right)+p L\left(U_{o}\right)+p[N(U)-f(r)]=0 .
$$

In equations (24) and 25 $p \in[0,1]$ is an embedding parameter, $u_{o}$ is an initial approximation for equation 19 , which satisfies the boundary conditions

Also, from equations 24) and 25 we will have

$$
\begin{gathered}
H(U, 0)=L(U)-L\left(U_{o}\right)=0, \\
H(U, 0)=A(U)-f(r)=0 .
\end{gathered}
$$

The changing process of $p$ from zero to unity is just that of $U(r, p)$ from $u_{o}(r)$ to $u(r)$. This is referred to homotopy in topology. Using the embedding parameter $p$ as a small parameter, the solution of equations (24) and (25) can be assumed to be written as a power series in $p$ as given in (28):

$$
U=U_{o}+p U_{1}+p^{2} U_{2}+\cdots .
$$

It should be pointed out that of all the values of $p$ between 0 and $p=1$ produces the best result. Therefore, setting $p=1$, results in the approximation solution of equation 21):

$$
u=\lim _{p \rightarrow 1} U=U_{o}+U_{1}+U_{2}+\cdots .
$$

The basic idea expressed above is a combination of homotopy and perturbation method. Hence, the method is called homotopy perturbation method (HPM), which has eliminated the limitations of the traditional perturbation methods. On the other hand, this technique can have the full advantages of the traditional perturbation techniques. The series 29 is convergent for most cases. 


\subsection{Application of the homotopy perturbation method to the present prob-}

lem. According to the homotopy perturbation method (HPM), one can construct an homotopy for equation 190 as

$$
H(\theta, p)=(1-p)\left[\frac{d^{2} \theta}{d X^{2}}\right]+p\left[\frac{d^{2} \theta}{d X^{2}}-S_{h} \theta^{2}-M_{a}^{2} \theta+M^{2} G(1+\gamma \theta)\right]
$$

where $p \in[0,1]$ is an embedding parameter. For $p=0$ and $p=1$ we have

$$
\theta(X, 0)=\theta_{0}(X), \quad \theta(X, 1)=\theta_{0}(X)
$$

We shall assume that the solution of equation 19 can be given in a series of the form

$$
\theta(X)=\theta_{0}(X)+p \theta_{1}(X)+p^{2} \theta_{2}(X)+p^{3} \theta_{2}(X)+\cdots=\sum_{i=0}^{n} p^{i} \theta_{i}(X) .
$$

On substituting equation $\sqrt{32}$ ) and into equation (30) and expanding the equation and collecting all terms with the same order of together, the resulting equation appears in form of polynomial in $p$. On equating each coefficient of the resulting polynomial in $p$ to zero, we arrive at a set of differential equations and the corresponding boundary conditions as

$$
\begin{aligned}
& p^{0}: \frac{d^{2} \theta_{0}}{d X^{2}}(X)=0, \quad \theta_{0}(0)=1, \quad \theta_{0}^{\prime}(1)=0 ; \\
& p^{1}: \frac{d^{2} \theta_{1}}{d X^{2}}+M^{2} G \gamma \theta_{0}-S_{h} \theta_{0}^{2}-M_{a}^{2} \theta_{0}+M^{2} G=0, \quad \theta_{1}(0)=0, \quad \theta_{1}^{\prime}(1)=0 ; \\
& p^{2}: \frac{d^{2} \theta_{2}}{d X^{2}}+M^{2} G \gamma \theta_{1}-S_{h} \theta_{0} \theta_{1}-M_{a}^{2} \theta_{1}=0, \quad \theta_{2}(0)=0, \quad \theta_{2}^{\prime}(1)=0 ; \\
& p^{3}: \frac{d^{2} \theta_{3}}{d X^{2}}+M^{2} G \gamma \theta_{2}-S_{h} \theta_{1}^{2}-2 S_{h} \theta_{0} \theta_{2}-M_{a}^{2} \theta_{1}+M^{2} G=0, \\
& \theta_{3}(0)=0, \quad \theta_{3}^{\prime}(1)=0 ; \\
& \theta_{4}(0)=0, \quad \theta_{4}^{\prime}(1)=0 ; \\
& p^{5}: \frac{d^{2} \theta_{4}}{d X^{2}}-M_{a}^{2} \theta_{3}-2 S_{h} \theta_{1} \theta_{2}-2 S_{h} \theta_{0} \theta_{3}+M^{2} G \gamma \theta_{3}=0, \\
& \theta_{5}(0)=0 \quad \theta_{5}^{\prime}(1)=0 ; \\
& p^{6}: \frac{d^{2} \theta_{6}}{d X^{2}}+M^{2} G \gamma \theta_{5}-2 S_{h} \theta_{0} \theta_{5}-2 S_{h} \theta_{1} \theta_{4}-M_{a}^{2} \theta_{5}-2 S_{h} \theta_{2} \theta_{3}=0, \\
& \theta_{6}(0)=0, \quad \theta_{6}^{\prime}(1)=0 ; \\
& \theta_{8}(0)=0, \quad \theta_{8}^{\prime}(1)=0 ; \\
& \theta_{7}(0)=0, \quad \theta_{7}^{\prime}(1)=0 ; \\
& p^{7}: \frac{d^{2} \theta_{7}}{d X^{2}}+M^{2} G \gamma \theta_{6}-2 S_{h} \theta_{1} \theta_{5}-2 S_{h} \theta_{0} \theta_{6}-M_{a}^{2} \theta_{6}-2 S_{h} \theta_{2} \theta_{4}=0, \\
& p^{8}: \frac{d^{2} \theta_{8}}{d X^{2}}+M^{2} G \gamma \theta_{7} \theta_{4}=0,2 S_{h} \theta_{3} \theta_{4}-2 S_{h} \theta_{1} \theta_{6}-M_{a}^{2} \theta_{7}-2 S_{h} \theta_{0} \theta_{7}-2 S_{h} \theta_{2} \theta_{5}=0 \\
&
\end{aligned}
$$




$$
\begin{aligned}
& p^{9}: \frac{d^{2} \theta_{9}}{d X^{2}}-2 S_{h} \theta_{0} \theta_{8}-2 S_{h} \theta_{2} \theta_{6}+M^{2} G \gamma \theta_{8}-S_{h} \theta_{4}^{2}-2 S_{h} \theta_{3} \theta_{5}- \\
&-2 S_{h} \theta_{1} \theta_{7}-M_{a}^{2} \theta_{8}=0, \quad \theta_{9}(0)=0, \quad \theta_{9}^{\prime}(1)=0 .
\end{aligned}
$$

On solving the above equations $(33)-(42)$ we arrive at

$$
\begin{aligned}
\theta_{0}(X) & =1 \\
\theta_{1}(X) & =\frac{1}{2}\left[M_{a}^{2}-M^{2} G(1+\gamma)+S_{h}\right]\left(X^{2}-1\right), \\
\theta_{2}(X) & =\frac{1}{24}\left[M_{a}^{2}-M^{2} G(1+\gamma)+S_{h}\right]\left(M_{a}^{2}+2 S_{h}-M^{2} G \gamma\right)\left(X^{4}-6 X^{2}+5\right), \\
\theta_{3}(X) & =\left\{\frac{S_{h}}{4}\left[M_{a}^{2}-M^{2} G(1+\gamma)+S_{h}\right]^{2}+\right. \\
& \left.+\frac{1}{24}\left[M_{a}^{2}-M^{2} G(1+\gamma)+S_{h}\right]\left(M_{a}^{2}+2 S_{h}-M^{2} G \gamma\right)^{2}\right\} \frac{X^{6}}{30}- \\
& -\left\{\frac{S_{h}}{2}\left[M_{a}^{2}-M^{2} G(1+\gamma)+S_{h}\right]^{2}+\right. \\
& \left.+\frac{1}{4}\left[M_{a}^{2}-M^{2} G(1+\gamma)+S_{h}\right]\left(M_{a}^{2}+2 S_{h}-M^{2} G \gamma\right)^{2}\right\} \frac{X^{4}}{12}+ \\
& +\left\{\frac{S_{h}}{4}\left[M_{a}^{2}-M^{2} G(1+\gamma)+S_{h}\right]^{2}+\right. \\
& \left.+\frac{5}{24}\left[M_{a}^{2}-M^{2} G(1+\gamma)+S_{h}\right]\left(M_{a}^{2}+2 S_{h}-M^{2} G \gamma\right)^{2}\right\} \frac{X^{2}}{2}- \\
& -\frac{11 S_{h}}{120}\left[M_{a}^{2}-M^{2} G(1+\gamma)+S_{h}\right]^{2}+ \\
+ & \frac{61}{720}\left[M_{a}^{2}-M^{2} G(1+\gamma)+S_{h}\right]\left(M_{a}^{2}+2 S_{h}-M^{2} G \gamma\right)^{2} .
\end{aligned}
$$

In the same manner expressions can be obtained for $\theta_{4}(X), \theta_{5}(X), \theta_{6}(X), \theta_{7}(X)$, $\theta_{8}(X), \theta_{9}(X)$. However they are too large expressions to be included in this paper.

From the definition, the solution of equation 19 in HPM domain is

$$
\begin{aligned}
\theta(X)=\theta_{0}(X)+p \theta_{1}(X)+ & p^{2} \theta_{2}(X)+p^{3} \theta_{3}(X)+p^{4} \theta_{4}(X)+p^{5} \theta_{5}(X)+ \\
& +p^{6} \theta_{6}(X)+p^{7} \theta_{7}(X)+p^{8} \theta_{8}(X)+p^{9} \theta_{9}(X)+\cdots
\end{aligned}
$$

It should be pointed out that of all the values of $p$ between 0 and $1, p=1$ produces the best result. Therefore, setting $p=1$ results in the approximation solution of equation 19):

$$
\begin{aligned}
\theta(X)=\lim _{p \rightarrow 1} \theta(X)=\theta_{0}(X)+\theta_{1}(X) & +\theta_{2}(X)+\theta_{3}(X)+\theta_{4}(X)+\theta_{5}(X)+ \\
& +\theta_{6}(X)+\theta_{7}(X)+\theta_{8}(X)+\theta_{9}(X)+\cdots
\end{aligned}
$$

After substituting equations (43)- 47 we have 


$$
\begin{aligned}
\theta(X) & =1-\frac{1}{2}\left[M_{a}^{2}-M^{2} G(1+\gamma)+S_{h}\right]\left(X^{2}-1\right)+ \\
& +\frac{1}{24}\left[M_{a}^{2}-M^{2} G(1+\gamma)+S_{h}\right]\left(M_{a}^{2}+2 S_{h}-M^{2} G \gamma\right)\left(X^{4}-6 X^{2}+5\right)+ \\
& +\left\{\frac{S_{h}}{4}\left[M_{a}^{2}-M^{2} G(1+\gamma)+S_{h}\right]^{2}+\right. \\
& \left.+\frac{1}{24}\left[M_{a}^{2}-M^{2} G(1+\gamma)+S_{h}\right]\left(M_{a}^{2}+2 S_{h}-M^{2} G \gamma\right)^{2}\right\} \frac{X^{6}}{30}- \\
& -\left\{\frac{S_{h}}{2}\left[M_{a}^{2}-M^{2} G(1+\gamma)+S_{h}\right]^{2}+\right. \\
& \left.+\frac{1}{4}\left[M_{a}^{2}-M^{2} G(1+\gamma)+S_{h}\right]\left(M_{a}^{2}+2 S_{h}-M^{2} G \gamma\right)^{2}\right\} \frac{X^{4}}{12}+ \\
& +\left\{\frac{S_{h}}{4}\left[M_{a}^{2}-M^{2} G(1+\gamma)+S_{h}\right]^{2}+\right. \\
& \left.+\frac{5}{24}\left[M_{a}^{2}-M^{2} G(1+\gamma)+S_{h}\right]\left(M_{a}^{2}+2 S_{h}-M^{2} G \gamma\right)^{2}\right\} \frac{X^{2}}{2}- \\
& -\frac{11 S_{h}}{120}\left[M_{a}^{2}-M^{2} G(1+\gamma)+S_{h}\right]^{2}+ \\
& +\frac{61}{720}\left[M_{a}^{2}-M^{2} G(1+\gamma)+S_{h}\right]\left(M_{a}^{2}+2 S_{h}-M^{2} G \gamma\right)^{2} .
\end{aligned}
$$

As regards the parameters $S_{h}, M_{a}, M$ and $G$ the reader is referred to equations (20b).

\section{Results And Discussion}

Figure 2 shows the effects of porous parameter or porosity on the temperature distribution in the porous fin. From the figures, as the porosity parameter increases, the temperature decreases rapidly and the rate of heat transfer (the convective-radiative heat transfer) through the fin increases as the temperature in the fin drops faster (becomes steeper, reflecting high base heat flow rates) as depicted in the figures. The rapid decrease in fin temperature due to increase in the porosity parameter occurs because as the porosity parameter, Raleigh number increases, the permeability of the porous fin increases and therefore the ability of the working fluid to penetrate through the fin pores increases, the effect of buoyancy force increases and thus the fin convects more heat, the rate of heat transfer from the fin is enhanced and the thermal performance of the fin is increased. Therefore, increase in the porosity of the fin improves fin efficiency by increasing convection heat transfer.

Figure 3 show the effects of conduction-convection parameter on the temperature distribution in the fin. The figure depicts that as the conduction-convection parameter increases, the rate of heat transfer through the fin increases as the temperature in the fin drops faster (becomes steeper, reflecting high base heat flow rates) as depicted 


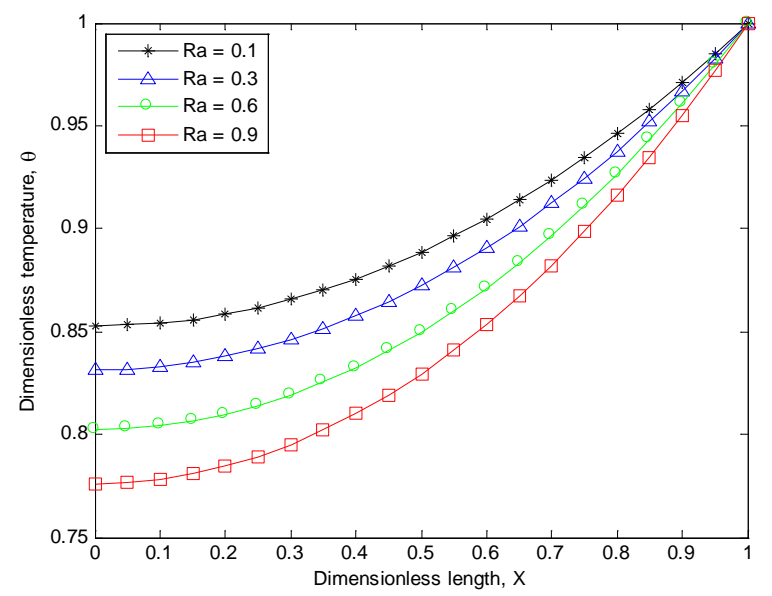

Figure 2. Dimensionless temperature distribution in the fin parameters for varying porous parameter when $R d=0.5, N c=0.6$, $N r=0.1, \varepsilon=0.8$ and $H a=0.7, Q=0$

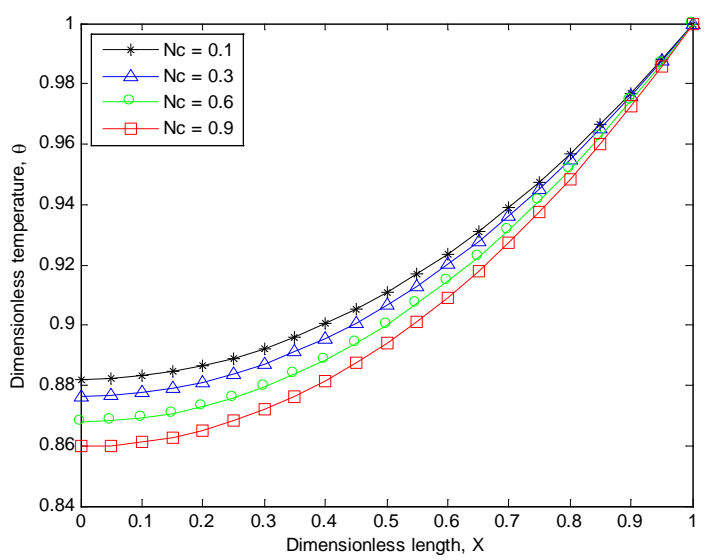

Figure 3. Dimensionless temperature distribution in the fin parameters for varying convection-conduction parameters when $R d=0.5$, $R a=0.3, N r=0.2, \varepsilon=0.7, Q=0$ and $H a=0.6$

in the figures. The profile has the steepest temperature gradient at lower value of the conduction-convection term, but its much higher value gotten from the lower value of thermal conductivity than the other values of $N c$ in the profiles produces a lower heat-transfer rate. This shows that the thermal performance or efficiency of the fin is favoured at low values of convective parameter since the aim (high effective use of the fin) is to minimize the temperature decrease along the fin length, where the best 


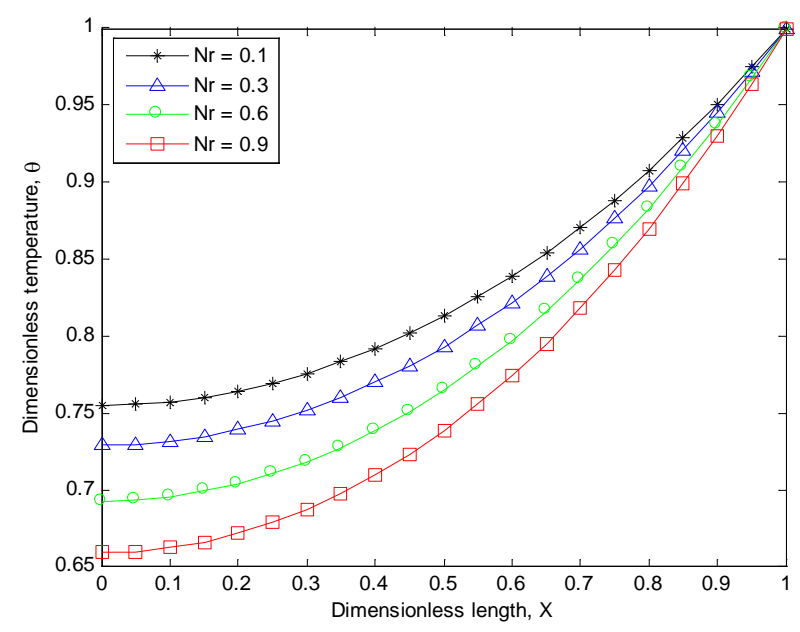

Figure 4. Dimensionless temperature distribution in the fin parameters for varying radiation-conduction parameter when $R d=0.8$, $R a=0.7, N c=0.5, \varepsilon=0.2, Q=0$ and $H a=0.3$

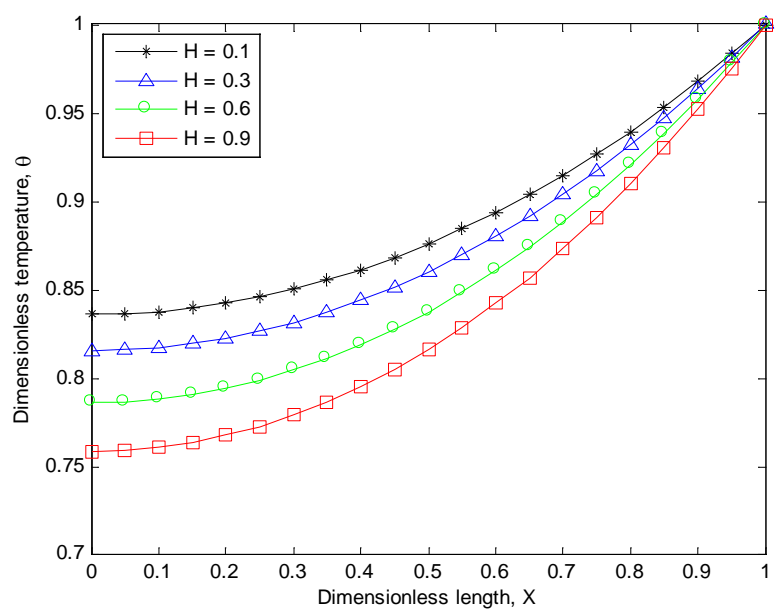

Figure 5. Dimensionless temperature distribution in the fin parameters for varying Hartman number (magnetic parameter), when $R d=0.6, R a=0.5, N c=0.1, Q=0, N r=0.7$ and $\varepsilon=0.4$

possible scenario is when $T=T_{b}$ everywhere. It must be pointed out that a small value of $\mathrm{M}$ corresponds to relatively short and thick fins of poor thermal conductivity and high value of $M$ implies a long fin or fin with low value of thermal conductivity. Since the thermal performance or efficiency of the fin is favoured at low values of convective fin parameter, very long fins are to be avoided in practice. 


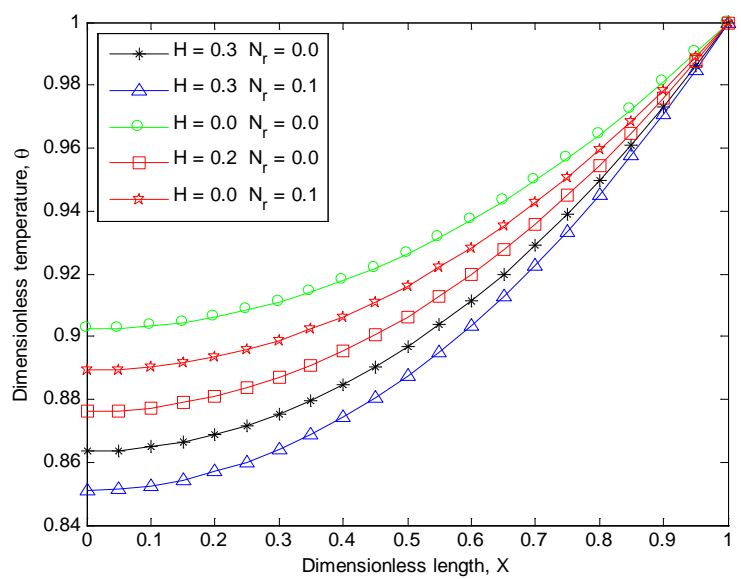

Figure 6. Dimensionless temperature distribution in the fin parameters for varying Hartman parameters and surface-ambient radiation parameters, when $R d=0.5, R a=0.4, N c=0.3, Q=0$ and $\varepsilon=0.1$

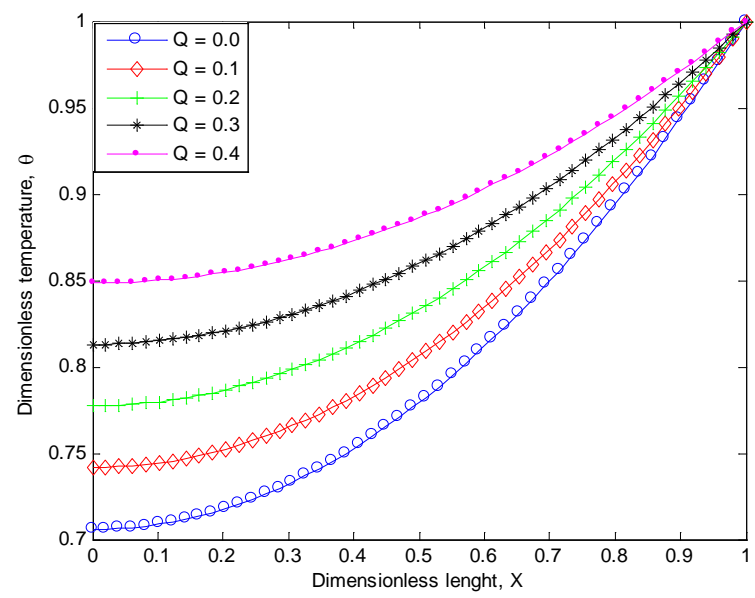

Figure 7. Dimensionless temperature distribution in the fin parameters for varying internal heat generation parameters, when $R d=0.25$, $R a=2.0, N c=1.0, N r=0.8, \tilde{a}=0.2, H=0.4$ and $\varepsilon=0.2$

The effects of conduction-radiation parameter are shown in Figure 4 The figure shows that with increase in the conduction-radiation parameter, the rate of heat transfer through the fin increases.

Figure 5 shows effects of the magnetic parameter, Hartman number on the temperature distribution in the porous fin. The figure depicts that the induced magnetic field in the fin can improve heat transfer through porous fins. This is also depicted in Figure 6 and it is also shown that conduction-radiation parameter increases the thermal performance of the fin. 


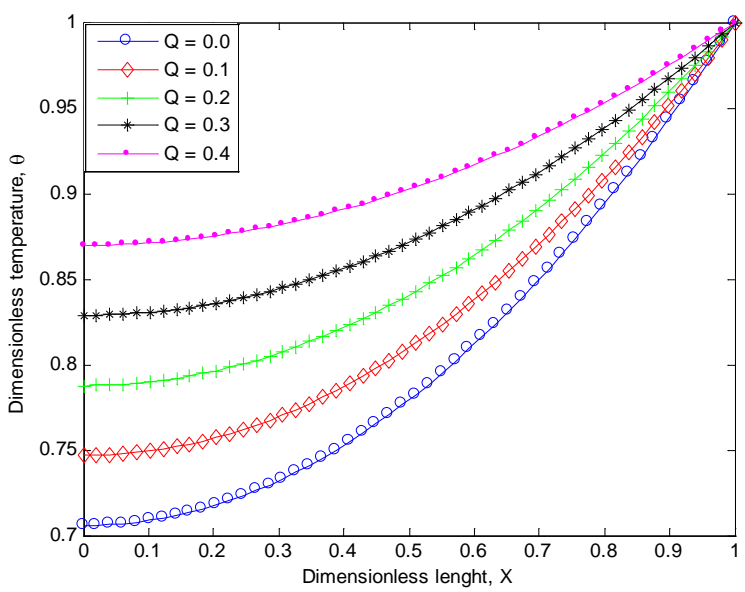

Figure 8. Dimensionless temperature distribution in the fin parameters for varying internal heat generation parameters, when $R d=0.25$, $R a=2.0, N c=1.0, N r=0.8, \tilde{a}=0.4, H=0.4$ and $\varepsilon=0.2$

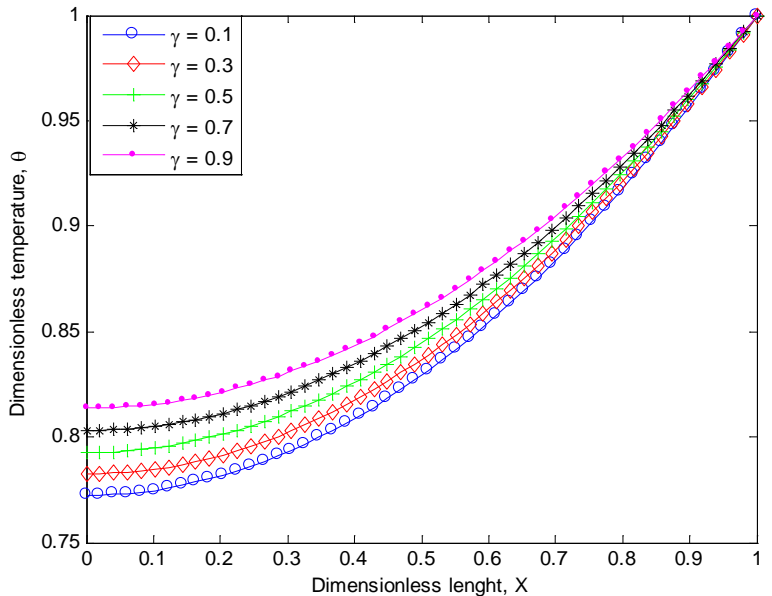

Figure 9. Dimensionless temperature distribution in the fin parameters for varying temperature-dependent internal heat generation parameters, when $R d=0.25, R a=2.0, N r=0.8, N c=1.0, H=0.4$, $Q=0.2$ and $\varepsilon=0.2$

From Figure 2 it is shown that increase in porosity, convective, radiative and magnetic parameters increases the rate of heat transfer from the fin and consequently improves the efficiency of the fin.

Figures 7 and 8 show the effects of internal heat generation parameter on the temperature distribution in the porous fin while Figures 9 and 10 depict the effects of 


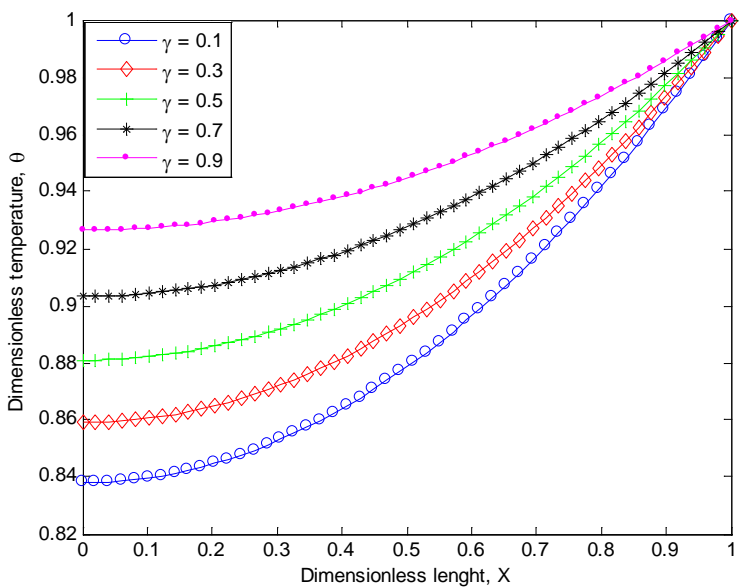

Figure 10. Dimensionless temperature distribution in the fin parameters for varying temperature-dependent internal heat generation parameters, when $R d=0.25, R a=2.0, N r=0.8, N c=1.0, H=0.4$, $Q=0.4$ and $\varepsilon=0.2$

Table 1. Comparison of results of NM and HPM for $\theta(X)$ and $R d=$ $0.5, \varepsilon=0.1, R a=0.4, N c=0.3, Q=0, N r=0.2, H=0.1$

\begin{tabular}{|c|c|c|c|}
\hline $\mathrm{X}$ & $\mathrm{NM}$ & HPM & $\begin{array}{c}\text { Absolut } \\
\text { Error }\end{array}$ \\
\hline \hline 0.00 & 0.863499231 & 0.863499664 & 0.000000433 \\
\hline 0.05 & 0.863828568 & 0.863829046 & 0.000000478 \\
\hline 0.10 & 0.864817090 & 0.864817539 & 0.000000449 \\
\hline 0.15 & 0.866466182 & 0.866465743 & 0.000000439 \\
\hline 0.20 & 0.868776709 & 0.868776261 & 0.000000448 \\
\hline 0.25 & 0.871751555 & 0.871751104 & 0.000000451 \\
\hline 0.30 & 0.875393859 & 0.875393404 & 0.000000455 \\
\hline 0.35 & 0.879707472 & 0.879707010 & 0.000000462 \\
\hline 0.40 & 0.884696967 & 0.884696500 & 0.000000467 \\
\hline 0.45 & 0.890367650 & 0.890367181 & 0.000000469 \\
\hline 0.50 & 0.896725569 & 0.896725096 & 0.000000473 \\
\hline 0.55 & 0.903777531 & 0.903777060 & 0.000000471 \\
\hline 0.60 & 0.911531120 & 0.911530658 & 0.000000462 \\
\hline 0.65 & 0.919994710 & 0.919994259 & 0.000000451 \\
\hline 0.70 & 0.929177488 & 0.929177056 & 0.000000432 \\
\hline 0.75 & 0.939089476 & 0.939089079 & 0.000000397 \\
\hline 0.80 & 0.949741555 & 0.949741203 & 0.000000352 \\
\hline 0.85 & 0.961145491 & 0.961145189 & 0.000000302 \\
\hline 0.90 & 0.973313964 & 0.973313764 & 0.000000200 \\
\hline 0.95 & 0.986260599 & 0.986260549 & 0.000000005 \\
\hline 1.00 & 1.000000000 & 1.000000000 & 0.000000000 \\
\hline & & & \\
\end{tabular}


temperature-dependent internal heat generation parameter on the temperature distribution in the fin. From the figures, as the internal heat generation parameters increase the temperature gradient of the fins decreases and consequently, the rate of heat transfer in the fin decreases. It should be stated that fins with porous material give superior performance with a significant reduction in weight compared with solid metal fins because of low thermal conductivity and the large area of the material that comes in contact with the cooling fluid.

Table 1 shows a comparison of results and the errors in the method used in this study. It could be inferred from the table that the HPM is highly accurate and agrees very well with the numerical method.

\section{Conclusion}

In this work, thermal performance in a porous fin temperature-dependent thermal properties and internal heat generation has been analyzed using the homotopy perturbation method. The developed symbolic heat transfer models were used to investigate the effects of various parameters on the thermal performance of the porous fin. Increasing the porosity increases the rate of heat transfer from the base of the fin and consequently improve the efficiency of the fin. Also, decreasing thermal conductivity parameter, results in increase in the rate of heat transfer from the base of the fin. The homotopy perturbation method used in the work was validated with the numerical method using Runge-Kutta method. The homotopy perturbation method results are in excellent agreement with results of the numerical method.

\section{NOMENCLATURE}

\section{Latin symbols}

$A_{c r} \quad$ cross sectional area of porous fin surface area, $\mathrm{m}^{2}$,

$B$ magnetic induction $(T)$,

$B_{o} \quad$ magnetic field intensity $(T)$,

$c_{p} \quad$ specific heat capacity, $J /(k g K)$,

Da Darcy number,

$E \quad$ electric field, $V / m$,

$g \quad$ acceleration due to gravity, $\mathrm{m} / \mathrm{s}^{2}$,

$h \quad$ heat transfer coefficient, $W m^{-2} k^{-1}$,

$h_{b} \quad$ heat transfer coefficient at the base of the fin, $W m^{-2} k^{-1}$,

$J_{c} \quad$ conduction current intensity $(A)$,

$k \quad$ thermal conductivity of the fin material, $W m^{-1} k^{-1}$,

$k_{b} \quad$ thermal conductivity of the fin material at the base of the fin, $W m^{-1} k^{-1}$,

$k_{\text {eff }} \quad$ effective thermal conductivity, $W m^{-1} k^{-1}$,

$K \quad$ permeability of the porous fin, $\mathrm{m}^{2}$,

$L \quad$ length of the fin, $m$,

$M \quad$ dimensionless thermo-geometric parameter,

$m$ mass flow rate of fluid passing through porous fin, $\mathrm{kg} / \mathrm{s}$,

$\mathrm{Nu} \quad$ Nusselt number, 
$N c \quad$ convective heat parameter,

$N r \quad$ radiative heat parameter,

$P \quad$ perimeter of the fin, $m$,

$q \quad$ internal heat generation, $W / m^{3}$,

$Q \quad$ dimensionless heat transfer rate per unit area,

$q_{b} \quad$ heat transfer rate per unit area at the base, $W / m^{2}$

$Q_{b} \quad$ dimensionless heat transfer rate at the base of porous fin,

$Q_{s} \quad$ dimensionless heat transfer rate the base in solid fin,

$R_{d} \quad$ Radiation-conduction parameter,

$R a$ Rayleigh number,

$S_{h} \quad$ porosity parameter,

$t \quad$ thickness of the fin, $m$,

$T$ fin temperature, $K$,

$T_{a} \quad$ ambient temperature, $K$,

$T_{b} \quad$ fin base temperature, $K$,

$v \quad$ average velocity of fluid passing through porous fin, $\mathrm{m} / \mathrm{s}$,

$V \quad$ particle's velocity, $m / s$,

$x \quad$ axial length measured from fin tip, $m$,

$X \quad$ dimensionless length of the fin,

$W \quad$ width of the fin, $m$.

\section{Greek symbols}

$\beta \quad$ thermal conductivity parameter or non-linear parameter,

$\beta^{\prime} \quad$ coefficient of thermal expansion $\left(K^{-1}\right)$,

$\tilde{\varepsilon} \quad$ pores parameter,

$\phi \quad$ porosity or void ratio,

$\delta \quad$ fin thickness at its base, $m$,

$\delta_{b} \quad$ fin thickness at its base, $m$,

$\lambda$ dimensional internal heat generation parameter,

$\gamma \quad$ dimensionless internal heat generation parameter,

$\theta$ dimensionless temperature,

$\theta_{b} \quad$ dimensionless temperature at the base of the fin,

$\eta \quad$ efficiency of the fin,

$v \quad$ kinematic viscosity, $\mathrm{m}^{2} / \mathrm{s}$,

$\rho \quad$ density of the fluid, $\mathrm{kg} / \mathrm{m}^{3}$,

$\sigma \quad$ Stefan-Boltzmann constant,

$\sigma_{e} \quad$ particle charge, $C$.

\section{REFERENCES}

1. Kiwan, S. and Al-NimR, A.: Using porous fins for heat transfer enhancement. ASME Journal of Heat Transfer, 123, (2001), 790-795.

2. KiwAn, S.: Effect of radiative losses on the heat transfer from porous fins. International Journal of Thermal Sciences, 46, (2007), 1046-1055

3. Kiwhn, S.: Thermal analysis of natural convection porous fins. Transport in Porous Media, 67, (2007), 17-29. 
4. Kiwan, S. and Zeitoun, O.: Natural convection in a horizontal cylindrical annulus using porous fins. International Journal of Heat and Fluid Flow, 18(5), (2008), 618-634.

5. Gorla, R. S. and BAKIER, A. Y.: Thermal analysis of natural convection and radiation in porous fins. International Communications in Heat and Mass Transfer, 38, (2011), 638-645.

6. Kundu, B. and Bhanji, F.: An analytical prediction for performance and optimum design analysis of porous fins. International Journal of Refrigeration, 34, (2011), 337352.

7. Kundu, B., Bhanji, F. and Lee, K. S.: A model on the basis of analytics for computing maximum heat transfer in porous fins. International Journal of Heat and Mass Transfer, 55(25-26), (2012), 7611-7622.

8. Taklifi, A., Aghanajafi, C. and Akrami, H.: The effect of MHD on a porous fin attached to a vertical isothermal surface. Transport in Porous Media, 85, (2010), 215-31.

9. Bhanja, D. and Kundu, B.: Thermal analysis of a constructal T-shaped porous fin with radiation effects. International Journal of Refrigeration, 34, (2011), 1483-1496.

10. Kundu B.: Performance and optimization analysis of SRC profile fins subject to simultaneous heat and mass transfer. International Journal of Heat and Mass Transfer, 50, (2007), 1545-1558.

11. Gorla, R., Darvishi, R. S. and Khani, M. T.: Effects of variable thermal conductivity on natural convection and radiation in porous fins. International Communications in Heat and Mass Transfer, 38, (2013), 638-645.

12. Saedodin, S. and Shahbabaei, M.: Thermal analysis of natural convection in porous fins with homotopy perturbation method (HPM). Arabian Journal for Science and Engineering, 38, (2013), 2227-2231.

13. Darvishi, M. T., Gorla, R. S. R., Gorla, R. and Aziz, A.: Thermal performance of a porus radial fin with natural convection and radiative heat losses. Thermal Science, 19(2), (2015), 669-678.

14. Moradi, A., Hayat, T. and Alsaedi, A.: Convective-radiative thermal analysis of triangular fins with temperature-dependent thermal conductivity by DTM. Energy Conversion and Management, 77, (2014), 70-77.

15. Ha, H. Ganji, D. D. and AbBasi, M.: Determination of temperature distribution for porous fin with temperature-dependent heat generation by homotopy analysis method. Journal of Applied Mechanical Engineering, 4(1), (2005), 1-5.

16. Hoshyar, H. A., Rahimipetroudi, I., Ganji, D. D. and Majidian, A. R.: Thermal performance of porous fins with temperature-dependent heat generation via homotopy perturbation method and collocation method. Journal of Applied Mathematics and Computational Mechanics, 14(4), (2015), 53-65.

17. Hatami, D. and Ganji, D. D.: Thermal performance of circular convective-radiative porous fins with different section shapes and materials. Energy Conversion and Management, 76, (2013), 185-193.

18. Hatami, D. and Ganji, D. D.: Thermal behavior of longitudinal convective-radiative porous fins with different section shapes and ceramic materials ( $\mathrm{SiC}$ and $\mathrm{Si3N} 4)$. International of J. Ceramics International, 40, (2014), 6765-6775. 
19. Rostamiyan, Y., Ganji, D. D., Petroudi, I. R. and Nejad, K. M.:. Analytical investigation of nonlinear model arising in heat transfer through the porous fin. Thermal Science, 18(2), (2014), 409-417.

20. Ghasemi, S. E., Valipour, P., Hatami, M. and Ganji D. D.: Heat transfer study on solid and porous convective fins with temperature-dependent heat -generation using efficient analytical method. Journal of Central South University of Technology, 21, (2014), 4592-4598.

21. He, J. H.: Homotopy perturbation technique. Computer Methods in Applied Mechanics and Engineering, 178, (1999), 257-262.

22. He, J. H.: New interpretation of homotopy perturbation method. International Journal of Modern Physics B, 20, (2006), 2561-2568.

23. He, J. H.: A coupling method of homotopy technique and perturbation technique for nonlinear problems. International Journal of Non-Linear Mechanics, 35, (2000), 37-43.

24. HE, J. H.: Some asymptotic methods for strongly nonlinear equations. International Journal of Modern Physics B, 20, (2006), 1141-1199.

25. He, J. H.: New perturbation technique which is also valid for large parameters. Journal of Sound and Vibration, 229, (2000), 1257-1263. 